\title{
Endothelial dysfunction: the role and impact of the renin-angiotensin system
}

\author{
Thomas F Lüscher
}

Division of Cardiology, Zurich, CH-8091

Zurich, Switzerland T F Lüscher

Correspondence to: Professor Lüscher Universitätsspital

A complex chain of events leads to the development of end stage heart disease (fig 1). It starts with seemingly normal subjects with certain risk factors, such as high cholesterol, hypertension, and diabetes. Atherosclerosis is the final common pathway, affecting blood vessels in the brain, the coronary circulation, and the peripheral circulation. What determines the prognosis for these patients is not the presence of myocardial ischaemia or angina pectoris, but the development of myocardial infarction-a complex disease involving coronary thrombosis, plaque rupture and vasospasm. As cardiac remodelling occurs in heart failure, other mechanisms also come into play. Throughout these stages of heart failure devel-

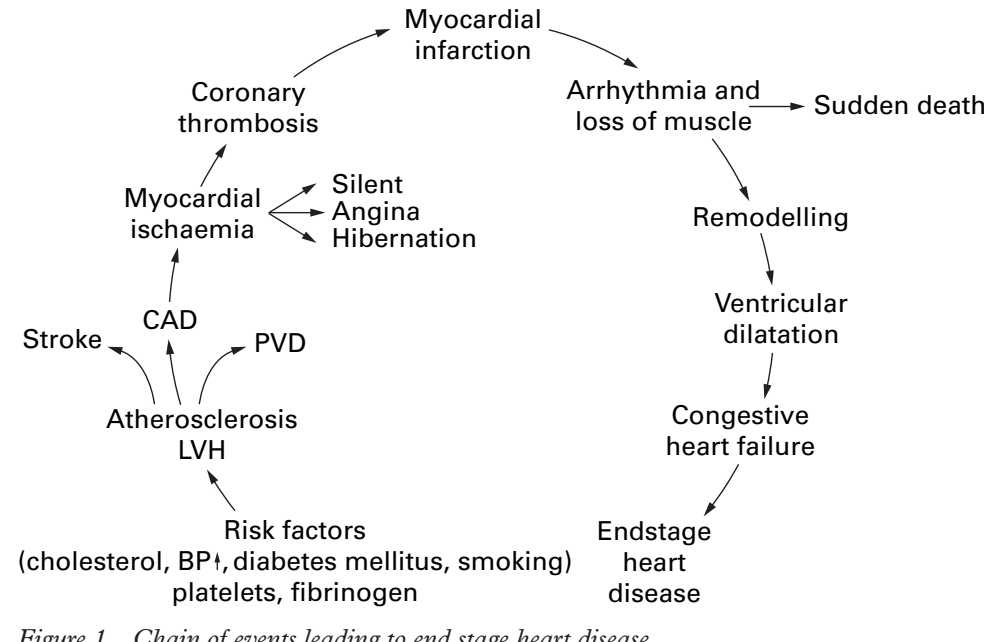

Figure 1 Chain of events leading to end stage heart disease.

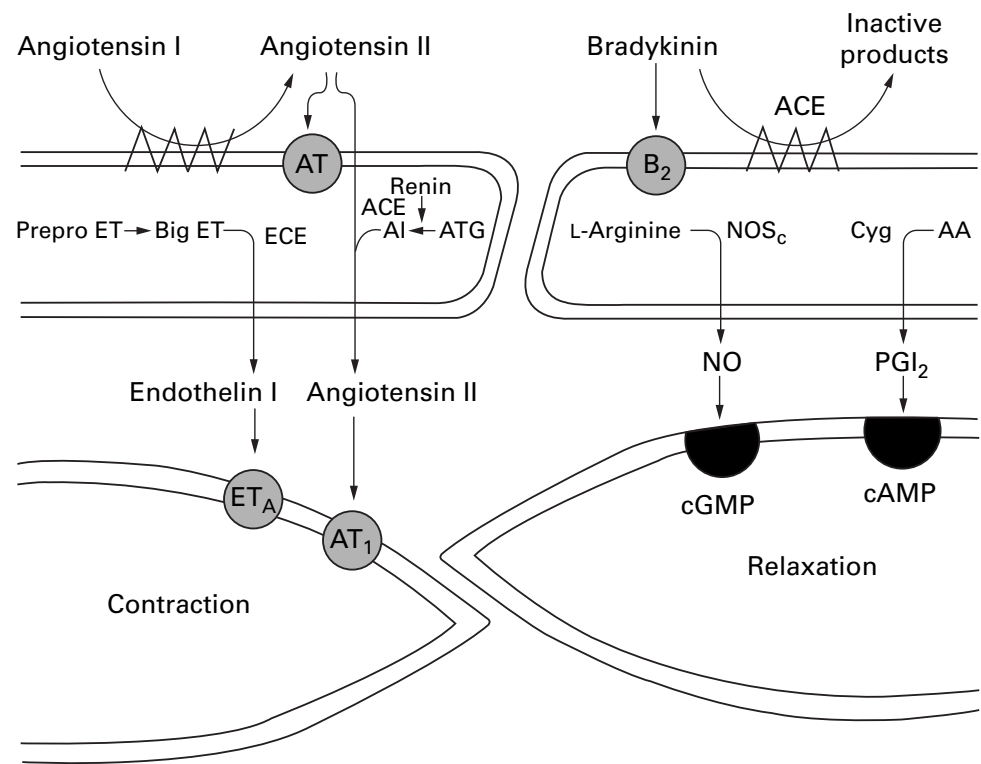

Figure 2 The endothelium plays an important role in the control of the vascular system. opment, the renin-angiotensin system plays an important role and provides a valuable target for therapeutic intervention.

Ten to 20 years ago the cardiovascular system was thought to be controlled by circulating factors such as the reninangiotensin system and the sympathetic nervous system which were able to regulate the heart, the kidney, and the blood vessels. More recently, however, it has become evident that the blood vessel itself plays an important role that involves many factors including nitric oxide, which is a vasodilator, and endothelin, a vasoconstrictor. In fact, the endothelium is altered morphologically as a result of coronary artery disease.

\section{Renin-angiotensin system and the endothelium}

Interestingly, angiotensin converting enzyme (ACE) is expressed at the endothelial cell membrane. As angiotensin II is created it may activate endothelial receptors or those expressed on smooth muscle cells. The receptors found on the endothelium are linked to the production of endothelin, a coagulation factor, and many other molecules including free radicals. The action of angiotensin II on smooth muscle cells produces contraction and also proliferation. These actions are supported by endothelin, which is itself stimulated by angiotensin II (fig 2).

\section{NITRIC OXIDE}

ACE inhibitors have several properties that may increase the bioavailability of endothelium derived nitric oxide - an important antiatherogenic molecule. For example, ACE inhibitors block the breakdown of bradykinin and independently stabilise the activity of the bradykinin receptor, thereby increasing the activity of the nitric oxide pathway. This shifts the balance from vasoconstriction to vasodilation. This has been illustrated in a number of studies.

In an in vitro study looking at human mammary resistance arteries, the addition of angiotensin I resulted in contraction (as the vessel wall is capable of converting angiotensin I into angiotensin II via ACE). ${ }^{1}$ If a high concentration of ACE inhibitor was added to the preparation, the contraction was notably reduced. The addition of an angiotensin receptor antagonist reduced the contraction even more effectively.

In a study in which the human saphenous vein was constricted using norepinephrine (noradrenaline), it was found that increasing concentrations of bradykinin produced a dose dependent relaxation. ${ }^{2}$ Interestingly, when these vessels were treated with an ACE inhibi- 
tor, there was a pronounced enhancement of the effects of bradykinin.

This is also seen in the human coronary artery, which does not contain $\alpha$ receptors, so contraction was produced using prostaglandin. ${ }^{3}$ The addition of the ACE inhibitor lisinopril to a constricted vessel had no effect. However, if the experiment was repeated using bradykinin to produce a partial relaxation, lisinopril exerted a further dose dependent relaxation, which was absent in the preparation without endothelium.

Clearly, there is an important action of ACE inhibitors at the level of nitric oxide and vasodilation.

\section{ENDOTHELIN}

Almost 10 years ago it was discovered that if porcine aortic endothelial cells are treated with angiotensin II, there is a time dependent increase in messenger RNA for the vasoconstrictor endothelin. ${ }^{4}$

In addition, when human endothelial cells in culture were exposed to an increasing angiotensin II concentration, there was a dose dependent increase in endothelin concentrations. ${ }^{5}$ The dose range of angiotensin II used was low, and reflected that which occurs under physiological conditions.

Moreover, it can also be shown that this process is occurring in the vessel wall and is completely prevented by either an endothelin receptor antagonist or by the angiotensin II receptor antagonist, losartan. ${ }^{6}$

Interestingly, the increase in systolic blood pressure following infusion of angiotensin II can be partially reduced by endothelin antagonism. ${ }^{7}$ The effects of angiotensin II on blood pressure control are therefore partly mediated by endothelin.

\section{Endothelial dysfunction}

Endothelial dysfunction is considered to play an important role in the pathogenesis of vascular disease. The vascular endothelium not only serves as a passive barrier to diffusion between the circulating blood and underlying vascular smooth muscle, but also regulates vessel diameter, inhibits blood clot formation, and impairs the multiplication of cells involved in plaque formation.

PROLIFERATION AND MIGRATION

There are both antiproliferation and antimigration endothelial mediated mechanisms. Nitric oxide inhibits cell cycle regulatory proteins. Similarly, prostacyclin can have some antiproliferative and antimigratory effects.

In human aortic smooth muscle cells, the number of migrating cells increases as angiotensin II concentrations are increased within a very low concentration range. As concentrations become higher, this is reversed-a transforming growth factor- $\beta$ (TGF- $\beta$ ) mediated mechanism. ${ }^{8}$

Another study looked at the effects of endothelin and endothelin antagonists on the proliferation of various growth factors. ${ }^{9}$ Platelet derived growth factor (PDGF) was found to stimulate proliferation quite strongly causing an eightfold increase in human smooth muscle cells. The addition of very low concentrations of endothelin can markedly potentiate the effects of the growth factors, PDGF or angiotensin. Endothelin is therefore a growth modulator of other growth factors. Interestingly, an endothelin receptor antagonist can prevent this response.

\section{OXIDATIVE STRESS}

Oxidative stress is a hallmark of hypertension, hyperlipidaemia, diabetes and, particularly, atherosclerosis. In atherosclerosis, NADPH oxidase enzymes located in the endothelium are a source of superoxide. There is a pronounced increase in superoxide production in the presence of angiotensin. This increase cannot be obtained by norepinephrine even though it produces a similar increase in blood pressure. It is therefore a highly specific effect of angiotensin II, which is not pressure related and is blocked by an angiotensin receptor antagonist. Angiotensin II not only acts through the endothelin axis, but also increases oxidative stress - an important mediator in cardiovascular disease.

\section{ATHEROSCLEROSIS}

Atherosclerosis has three major groups of mechanisms:

- Vasoconstriction-abnormal vasoconstriction of the coronary arteries particularly during exposure to sympathetic drive, such as cold, pressure etc;

- Structural changes-within the human coronary artery (plaques);

- Thrombus formation-caused by activation of platelets and coagulation factors.

The endothelium plays a role via four different phases of the atherosclerosis process:

(1) Early on in the disease process there is abnormal vasomotion. This is related to an increased breakdown of nitric oxide, due to oxidant stress and the increased production of endothelin.

(2) There is a decrease in the production of nitric oxide and an increase in the production of oxygen, allowing monocytes to adhere.

(3) There is proliferation and migration of smooth muscle cells.

(4) Finally, there is thrombus activation.

\section{ACE inhibitors and endothelial}

\section{dysfunction}

The TREND trial evaluated the effects of the ACE inhibitor quinapril, on coronary endothelial activity in angiographically normal coronary arteries, in subjects with documented coronary artery disease. ${ }^{10}$ All patients underwent percutaneous transluminal coronary angioplasty (PTCA) at the time of the first investigation and acetylcholine tests.

In the placebo group all arteries constricted paradoxically to acetylcholine because the patients had coronary artery disease. The quinapril treated patients showed a major reduction in vasoconstriction in response to acetylcholine, which was dose dependent and more pronounced at the higher concentration 
Trial acronyms

TREND: Trial on Reversing Endothelial Dysfunction

HOPE: Heart Outcomes Prevention Evaluation study

SOLVD: Studies Of Left Ventricular Dysfunction

of acetylcholine. There was a major improvement in coronary vasomotion in the quinapril treated patients, indicating that the reninangiotensin system contributes to endothelial dysfunction in patients with coronary artery disease.

Interestingly, the SOLVD trial found that, compared to placebo, the ACE inhibitor enalapril reduced the incidence of myocardial infarction. ${ }^{11}$ Possibly, some of the effects of ACE inhibitors may be related to an improvement in endothelial function. The HOPE trial, which showed a significant reduction of morbidity and mortality in patients with maintained left ventricular function, provides further confirmation of this concept. ${ }^{12}$

\section{Conclusion}

Cardiovascular haemostasis is a complex process. While several years ago only the circulating renin-angiotensin system and sympathetic nervous system were considered, it is now known that the blood vessels also contribute to vascular disease. In fact, the endothelium is an important player because of the release of endothelin, a potent peptide closely linked to the renin-angiotensin and sympathetic nervous systems. Moreover, the enzyme regulating the renin-angiotensin system not only has effects at the level of the smooth muscle cells within the blood vessel wall, but also at the endothelium, by regulating the release of the protective substance, nitric oxide.

ACE inhibitors are valuable tools for interfering with this complex mechanism, but in the future other approaches will be discovered involving systems of the nitric oxide axis and the endothelin pressure system.

1 Tschudi MR, Lüscher TF. Characterization of contractile endothelin and angiotensin receptors in human resistance arteries: evidence for two endothelin and one angiotensin receptor. Biophys Biochem Res 1994;204:685-90.

2 Yang Z, Arnet U, von Segesser L, et al. Different effects of angiotensin-converting enzyme inhibition in human arteries and veins. F Cardiovasc Pharmacol 1993;22(suppl 5):1722 .

3 Auch Schwelk W, Bossaller C, Claus C, et al. Local potentiation of bradykinin-induced vasodilation by converting-enzyme inhibition in isolated coronary arteries. 7 Cardiovasc Pharmacol 1992;20(suppl 9):S62-7.

4 Yanagisawa M, Kurihara H, Kimura S, et al. A novel potent vasoconstrictor peptide produced by vascular endothelial cells. Nature 1988;332:411-15.

5 Lüscher TF, Barton M. Endothelin and endothelin receptor antagonists. Circulation In press.

6 Moreau P, d'Uscio LV, Shaw S, et al. Angiotensin II increases tissue endothelin and induces vascular hypertrophy in vivo: reversal by ETA-receptor antagonist. Circulation 1997;96:1593-7.

7 d'Uscio L, Shaw S, Barton M, et al. Losartan but not verapamil inhibits angiotensin II-induced tissue endothelial-1 increase: role of blood pressure and endothelial function. Hypertension 1998;31:1305-10.

8 Liu G, Espinosa E, Oemar BS, et al. Bi-modal effects of angiotensin II on migration of human and rat smooth muscle cells: direct stimulation and indirect inhibition via transforming growth factor beta-1. Arterioscler Thromb Vasc Biol 1997;17:1251-7.

9 Yang Z, Krasnici N, Lüscher TF. Endothelin-1 potentiates human smooth muscle cell growth to PDGF: effects of ETA and ETB receptor blockade. Circulation 1999;100:58.

10 Mancini GBJ, Henry GC, Macaya C, et al. Angiotensinconverting enzyme inhibition with quinapril improves endothelial vasomotor dysfunction in patients with coronary artery disease: the TREND (trial on reversing endotheial dysfunction) study. Circulation 1996; 94:258-65.

11 The SOLVD Investigators. Effect of enalapril on survival in patients with reduced left ventricular ejection fractions and congestive heart failure. N Engl F Med 1991;325:293-302.

12 The Heart Outcomes Prevention Evaluation Study Investigators. N Engl F Med 2000;342:145-53. 